NASA Technical Memorandum 106501

AIAA-94-0357

\title{
Simulation of Low-Density Nozzle Plumes in Non-Zero Ambient Pressures
}

Chan-Hong Chung and Kenneth J. De Witt

The University of Toledo

Toledo, Ohio

and

Robert M. Stubbs and Paul F. Penko

Lewis Research Center

Cleveland, Ohio

Prepared for the

32nd Aerospace Sciences Meeting and Exhibit

sponsored by the American Institute of Aeronautics and Astronautics

Reno, Nevada, January 10-13, 1994

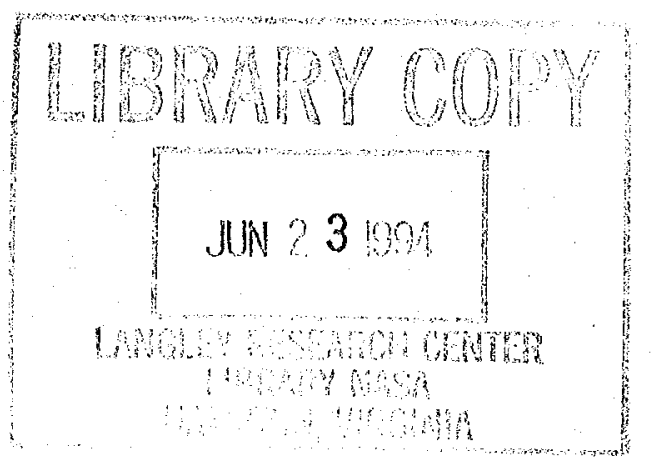




\section{,}

.

. 


\title{
Simulation of Low-Density Nozzle Plumes in Non-Zero Ambient Pressures
}

\author{
Chan-Hong Chung ${ }^{*}$ and Kenneth J. De Witt ${ }^{* *}$ \\ The University of Toledo \\ Toledo, Ohio 43606 \\ and \\ Robert M. Stubbs ${ }^{\dagger}$ and Paul F. Penko ${ }^{\ddagger}$ \\ National Aeronautics and Space administration \\ Lewis Research Center \\ Cleveland, Ohio 44135
}

\begin{abstract}
The direct simulation Monte-Carlo (DSMC) method was applied to the analysis of low-density nitrogen plumes exhausting from a small converging-diverging nozzle into finite ambient pressures. Two cases were considered that simulated actual test conditions in a vacuum facility. The numerical simulations readily captured the complicated flow structure of the overexpanded plumes adjusting to the finite ambient pressures, including Mach disks and barrelshaped shocks. The numerical simulations compared well to experimental data of Rothe.
\end{abstract}

\section{INTRODUCTION}

Low-thrust rocket engines which are used for stationkeeping, attitude and altitude control on various satellites and spacecraft have a significant impact on mission performance such as on-orbit lifetime, payload, and trip time. Another important factor affecting mission performance is contamination of sensitive instruments and system components that may be exposed to the thruster plumes both in the forward and backward flow directions. Hence, understanding of the detailed flow structure in and around low-thrust rocket nozzles is important not only for the accurate prediction of thrust and mass flow but also for the precise prediction of the plume flow regions.

Low-density flow through small nozzles expanding to vacuum has been examined previously in both experimental and numerical investigations, though, little experimental data are available and most data are for gross characteristics of nozzle performance such as thrust and discharge coefficients. Data that provides detailed information on internal flow structure was published by Rothe $[1,2]$ in which density and rotational temperature distributions were measured inside a small nozzle and in the

\footnotetext{
- NASA Resident Research Associate at Lewis Research Center.

- Professor, Member AIAA.

+ Chief, Computational Methods for Space Branch, Member AIAA.

₹ Aerospace Engineer, Member AIAA.
}

nozzle plume using the electron-beam fluorescence technique. Rothe's experiment was numerically simulated by Chung et al. [3] using a continuum code based on the Navier-Stokes equations and the direct simulation MonteCarlo (DSMC) method of Bird [4]. The simulation results were compared with Rothe's density and rotational temperature data at various locations inside the nozzle and at the nozzle exit plane. In this study, no consideration was given to simulation of the finite test-facility pressure. In somewhat related work, Pitot-pressure measurements and numerical simulations were made for a low-density nozzle and plume flow by Penko et al. [5] and Boyd et al. [6]. Comparisons were made between continuum and DSMC results and Pitot pressure measurements at the nozzle exit plane and various locations in the plume. Comparisons of continuum and DSMC results were also made for the flow inside the nozzle. In these works, test-facility pressures were quite low and, therefore, consideration was not given to numerical simulation of the actual ambient pressure. In other work, Campbell [7,8], Nelson and Doo [9], and Zelesnik et al. [10] also analyzed expanding low-density nozzle flows using the DSMC method and compared their results with experimental data.

The rocket engines under consideration typically have thrust levels under $100 \mathrm{mN}$, are physically small in size, and have relatively low operating pressures and mass flow. Reynolds numbers are low, on the order $10^{3}$, and rarefaction effects are significant. Under these conditions, the flow contains strong nonequilibrium effects, such as slip at a wall, from rapid expansion to near vacuum conditions. The flow transitions from continuum to freemolecular regimes. Conventional continuum gas dynamics may not be adequate to accurately analyze the flow and an approach based on kinetic theory may be required.

Of the various methods available for analysis of lowdensity gas flows, the DSMC method is most widely used and readily applicable. The DSMC method is a numerical simulation technique for solving the Boltzmann equation by modeling a real gas flow using a representative set of molecules. Theoretically, the DSMC method can be applied to any flow for which the Boltzmann equation is valid but intensive computational requirements generally restrict the use to near continuum and rarefied flows. 
Continuum methods are usually much more efficient than the DSMC method for higher density flows. Thus, in the analysis of flows which involve both continuum and rarefied regimes, it is reasonable to apply both methods. The simplest utilization of both methods is to solve the rarefied flow regime with the DSMC method using boundary conditions for the inflow surface obtained from the continuum method used to solve the continuum and near-continuum regime.

In this paper, the DSMC method is employed for the analysis of low-density nitrogen flows expanding through a small converging-diverging nozzle and into finite back pressures that simulate Rothe's experimental conditions. Special attention is given to the effect of the non-zero ambient pressure on the flow structure, both in the nozzle but especially in the plume, to simulate conditions often encountered in ground-based test facilities used to test small thrusters. In contrast to the behavior of low-density plumes expanding into a vacuum, the flow expanding into a region of finite pressure is often overexpanded with a more complicated flow pattern involving Mach disks and shock waves. Under these conditions, the supersonic flow can be confined to a narrow core, depending on the applied background pressure, and the sonic line may not intersect with the nozzle lip as it does with a vacuum ambient, in which case the external conditions can influence the internal flow through the thick subsonic region near the nozzle wall. These conditions are illustrated in the results from the numerical simulation and in comparison with Rothe's experimental data.

\section{PROBLEM STATEMENT}

Rothe's experiment [2] was chosen as a reference problem because of the availability of detailed measurements. Figure 1 illustrates the geometry of the nozzle used in Rothe's experiment and in the numerical simulation. The actual nozzle was made of graphite to reduce optical reflections and to minimize back-scattering and secondary emission of electrons. The subsonic and supersonic portions of the nozzle are cones having halfangles of $30^{\circ}$ and $20^{\circ}$, respectively, with longitudinal radii of curvature at the throat equal to $1 / 2$ of the throat radius. The area ratio at the exit based on the throat area is 66 . The shaded region in Fig. 1 indicates the domain of the DSMC simulation. The length of the curved contour downstream of the throat (IH) is about $0.5 \mathrm{~mm}$. The simulation domain extends from the throat to an axial distance $260 \mathrm{~mm}$ from the nozzle exit plane (AB), a radial distance $50 \mathrm{~mm}$ from the nozzle lip (GD), and an axial distance $20 \mathrm{~mm}$ from the nozzle exit plane into the backflow region (DE). The inflow boundary is located at the nozzle throat (OI). The boundary condition at the inflow boundary is obtained from a solution of the NavierStokes equations [3]. The radial boundary CE is located far enough from the axis so that extending it further radially does not result in any significant changes in the macroscopic flow variables in the plume. Along the boundaries $\mathrm{CE}$ and $\mathrm{EF}$ an equilibrium condition corresponding to the ambient gas is used as the boundary condition. Likewise, the downstream boundary BC is located far enough from the nozzle exit so that extending it further downstream does not result in any significant changes in the macroscopic flow variables in the near plume. Along the boundary BC, several boundary conditions are tested including an equilibrium condition corresponding to the ambient condition, a vacuum boundary condition, and an equilibrium condition corresponding to a profile extrapolated from the inside of the plume. The test gas is nitrogen with a stagnation temperature of $T_{n}=300 \mathrm{~K}$. The flow conditions are listed in Table 1. In the table, the throat Reynolds number, $R_{\mathrm{e}, \mathrm{t}}=2 \dot{m} / \pi \mu_{\mathrm{o}} R_{\mathrm{t}}$, is based on the viscosity at the stagnation chamber condition, $\mu_{0}$. Here the quantity $\dot{m}$ is the mass flow rate and $R_{\mathrm{t}}$ is the throat radius. The Knudsen number $K n$ is based on the throat diameter and the stagnation chamber condition.

\section{DSMC METHOD}

The DSMC code used in this study is based on the principles described by Bird [4], together with the variable hard sphere (VHS) model [11] as a molecular model and the no time counter (NTC) method [12] as a collision sampling technique. The code was developed to investigate various low-density flows of gas mixtures in arbitrarily shaped flow domains $[3,13,14]$. Details of the code may be found in Ref. 3. The execution speed of the code for the flow considered in this study, measured by CPU time/particle/timestep, is about $1.3 \mu$ s on a CRAY Y/MP. The flow domain consists of about 20,000 cells in 41 subregions. At the steady phase of the simulation, the total number of simulated molecules in the flow domain is about 2 million. The flow field is sampled every 5 timesteps during 20,000 timesteps after reaching the steady phase. The total CPU time required for the computation is about 19 hours on the CRAY Y/MP.

The VHS exponent, $\omega$, of nitrogen is chosen to be 0.24 with the reference molecular diameter of $4.07 \times 10^{-10} \mathrm{~m}$ at the reference temperature $273 \mathrm{~K}$ [11]. Chemical reactions and the vibrational mode are assumed to be frozen. For the calculation of rotational energy exchange between the colliding molecules, the Borgnakke-Larsen phenomenological model [15] is employed together with the temperature-dependent energy exchange probability of Boyd [16] modified by Chung et al. [3] to be consistent with the experimental data for the rotational relaxation of nitrogen obtained by various methods and compatible with the VHS model. A diffusely adiabatic wall with $10 \%$ thermal accommodation is assumed for the interaction between the gas molecules and the wall [3].

To assess the effect of backscattering of downstream molecules and reduce the number of simulated molecules, the gas is treated as a mixture consisting of two different nitrogen sources whose origins are the upstream reservoir and the downstream, respectively. A ratio of the actual to the simulated molecules for the nitrogen whose origin is the downstream is chosen to be 4 times larger than that for the nitrogen coming from the upstream reservoir. 


\section{RESULTS AND DISCUSSION}

To present the general idea of the over-expanded plumes and the overall structure of the flowfield, density and Mach number contours will be considered first. Figures 2 and 3 show density and Mach number contours for Case $I$ in which the stagnation pressure $P_{0}$ is $474 \mathrm{~Pa}$ and the ratio of stagnation to ambient pressure is $P_{\mathrm{o}} / P_{\mathrm{b}}=310$. The density is normalized by the background density $\rho_{b}$. In this case, the density decreases from the throat to the nozzle exit and approaches the background density in the plume. Around the nozzle lip, the density has a minimum value which is about $20 \%$ lower than the background density due to entrainment. The Mach number increases from the throat to the nozzle exit and then decreases as the flow adjusts to the ambient pressure. The maximum Mach number is about 3.7 and occurs just inside the nozzle near the exit. In contrast to the behavior observed in plumes expanding into a vacuum, the sonic line of the internal boundary layer does not intersect with the nozzle lip and is almost parallel to the nozzle surface. There is no sign of shock waves in this plume.

Figures 4 and 5 show density and Mach number contours for Case II in which the stagnation pressure is $1245 \mathrm{~Pa}$ and pressure ratio is $P_{\mathrm{o}} / P_{\mathrm{b}}=325$. Again, the densify is normalized by the background density $\rho_{b}$. In this case, the plume is overexpanded and a more complicated flow pattern exists with a barrel-shaped shock wave and a Mach disk. It can be seen that the supersonic portion of the flow is restricted to a narrow core and enveloped by the barrel-shaped shock wave. The flow in the core region is strongly decelerated at the Mach disk and there is a region of high density between the barrel-shaped shock and the plume boundary. Also, the plume shows a repetitive pattern of expansion, which is diffused far downstream.

The pressure distribution along the axis of the nozzle is depicted in Fig. 6, which shows the extent of overexpansion in the plume. It can be seen in the figure that even in Case I the flow is slightly overexpanded just outside the nozzle exit, where the minimum pressure is about $73 \%$ of the ambient pressure. However, the overexpansion is not strong enough to cause any significant disturbances in the flow. In case II, the centerline pressure at the exit is already $60 \%$ of the ambient pressure and drops to $33 \%$ of the ambient pressure at $25 \mathrm{~mm}$ downstream from the exit. Hence, back-pressure effects cause the flow to turn into the axis and produce the characteristic barrelshock configuration. Beyond the shock, the flow continues to expand. In this case, however, the expansion is not sufficiently strong to sustain the repetitive pattern of compression and expansion.

Consideration is now given to the effect of boundary conditions. For the simulation of nozzle flows with nonzero ambient pressures a supersonic outflow boundary condition, which is usually assumed along boundaries in the simulation of nozzle flows expanding into a vacuum, can not be used since the flow is not supersonic along the boundaries in the plume. The best way would be to extend the simulation domain sufficiently far so that the flow along the boundaries is not disturbed by the expanding plume and an equilibrium condition corresponding to the ambient gas may be used as the boundary condition, which, unfortunately, is computationally prohibitive. In the present study, the extension of the simulation domain to a radial distance $70 \mathrm{~mm}$ from the axis, and to an axial distance $20 \mathrm{~mm}$ from the nozzle exit plane into the backflow region, is found to be sufficient to impose the equilibrium condition corresponding to the far-field ambient condition. It is also found that the extension of the simulation domain to the downstream axial distance 260 $\mathrm{mm}$ from the nozzle exit plane is sufficient to resolve important characteristics of the plume.

Figure 7 shows the effect of the boundary condition along the downstream boundary on the centerline Mach number. Two distinctively different boundary conditions are imposed along the downstream boundary at $z=260$ $\mathrm{mm}$, including, an equilibrium condition corresponding to the ambient condition (dot-dashed line) and a vacuum boundary condition (dashed line). For a third condition, since the radial distribution of flow variables are very similar far downstream, profiles are extrapolated from the inside of the plume and an equilibrium condition corresponding to the profiles is employed as the boundary condition (solid line). For the sake of comparison, the result obtained by reducing the axial distance from the nozzle exit to the downstream boundary to about $180 \mathrm{~mm}$ and imposing an equilibrium condition corresponding to the ambient condition is also shown (dotted line). It can be seen that extremely different boundary conditions have no significant effect on the Mach number distribution in the near plume within $150 \mathrm{~mm}$ from the nozzle exit. Figure 8 shows the effect of the boundary condition on the radial translational temperature profile at the axial location $z=$ $150 \mathrm{~mm}$. Again it can be seen that extremely different boundary conditions along the downstream boundary have no significant effect on the radial temperature distribution in the near plume within $150 \mathrm{~mm}$ from the nozzle exit, where the most important and distinctive characteristics of the present overexpanded plume occurs. Although not shown, the effect of the boundary conditions on the other flow variables such as density, velocity, and rotational temperature is similar to those observed in Figs. 7 and 8.

The comparison of the simulation results with Rothe's experimental data[2] is made in Fig. 9 in which density profiles along the nozzle axis inside the nozzle and in the plume for Case I are shown. The densities are normalized by the ambient density $\rho_{b}$. The solid line is from the DSMC simulation with the ambient pressure and the filled circles represent the experimental data of Rothe. For comparison, a calculation made for the flow expanding into a vacuum is also shown in the figure and is represented by the dotted line. The simulation results indicate that for the two different ambient conditions, the density profiles along the axis show no significant differences inside the nozzle for the case where the stagnation pressure $P_{0}=474 \mathrm{~Pa}$. The simulation result with the non-zero ambient pressure shows good agreement with the experimental data both inside the nozzle and in the plume. Figure 10 shows the comparison of the simulation results with experimental data along the nozzle axis for Case II where $P_{0}=1245 \mathrm{~Pa}$. The density decreases from the throat to the nozzle exit before the shock occurs. The local minimum density is 
about 2.2 times the ambient density and occurs about 25 $\mathrm{mm}$ downstream from the exit. The local maximum density is about 6.4 times the ambient density and occurs about $60 \mathrm{~mm}$ downstream from the exit. The density profile along the axis with a non-zero ambient pressure and that for a vacuum ambient show no significant differences before the shock for this case. The simulation result with the non-zero ambient pressure shows good agreement with the experimental data across the shock except for the slight shift in the axial direction.

Comparison of calculated radial density profiles with Rothe's experimental data at various axial locations in the plume for Cases I and II are shown in Figs. 11 and 12, respectively. Again, the solid line is the simulation result with non-zero ambient pressure and the filled circles represent the experimental data. The densities are normalized by the ambient density $\rho_{b}$. The radial density profiles are arranged in a sequence corresponding to axial positions in the plume. At the lower stagnation pressure of $P_{\mathrm{o}}=474 \mathrm{~Pa}$, the density profiles have a single peak, which decrease both in the radial and axial directions. At the higher stagnation pressure of $P_{0}=1245 \mathrm{~Pa}$, the density profiles in the plume are double peaked and indicate the presence of a barrel-shaped shock. The barrel-shaped shock converges to a single density peak further downstream. In both cases, the jet is confined to a narrow core the diameter of which is about the same as the nozzle exit diameter. The simulation results show good agreement with the experimental data in both cases except for the region around the shock in the higher pressure case where a small difference in the axial location results in a significant difference in the profile as shown in Fig. 10.

The degree of thermal nonequilibrium in the plume is shown in Fig. 13 where both translational and rotational temperature distributions along the axis for Case II are plotted. For comparison, temperature distributions for the flow expanding into a vacuum are also shown in the figure. From the rapid expansion, the rotational temperature is higher than the translational temperature along the axis inside the nozzle and in the near plume. In the case of the flow expanding into a vacuum, the two temperatures are frozen far downstream. For the case of the non-zero ambient pressure, however, the translational temperature becomes higher than the rotational temperature across the shock wave from the rapid compression. Beyond the shock, the translational temperature is higher than the rotational temperature where the flow once again expands. Far downstream, the two temperatures become the same as the flow approaches equilibrium.

Finally, the effect of the non-zero ambient pressure on the exit plane density is shown in Figs. 14 and 15. In the figures, the quantity $R_{\mathrm{E}}$ is the exit radius of the nozzle. Figure 14 shows the exit plane density profiles for the stagnation pressure $P_{0}=1245 \mathrm{~Pa}$. Near the axis, the two densities are nearly the same and are not influenced by the external condition, while near the nozzle lip, the effect of the external condition becomes significant. At the nozzle wall, the density of the flow expanding into vacuum is $55 \%$ of that with the non-zero ambient pressure. Figure 15 shows the mole fraction of the background gas at the exit plane. It can be seen that near the axis the gas is composed of gas molecules exclusively coming from the upstream stagnation chamber. Near the nozzle lip, a significant number of gas molecules from the ambient have penetrated inside the nozzle. At the lip, the fraction of gas originating from the background gas is about $11 \%$ and $13 \%$ for Case I and II, respectively.

\section{CONCLUSIONS}

The flow structure in low-density plumes expanding through a small converging-diverging nozzle into a region of finite pressure is investigated using the direct simulation Monte-Carlo (DSMC) method based on molecular gas dynamics. This kind of flow structure has rarely been studied using the DSMC method. The calculated results show that the plume structure with a non-zero ambient pressure is substantially different from that of the flow expanding into a vacuum. The simulation results show good agreement with Rothe's experimental data measured at various axial and radial locations in the plume. It is shown that the DSMC method can successfully predict the complex flow structures such as a Mach disk and barrelshaped shock which are representative of overexpanded nozzle plumes.

\section{ACKNOWLEDGMENT}

Support for the first author (C. Chung) by the NASA Lewis Research Center, Cleveland, Ohio, under Grant NCC 3-171 is gratefully acknowledged. Dr. Nan-Suey Liu is the grant director.

\section{REFERENCES}

[1] Rothe, D. E., "Electron Beam Studies of Viscous Flow in Supersonic Nozzles," AIAA J., Vol. 9, No. 5, 1972, pp. 804-811.

[2] Rothe, D. E., Experimental Study of Viscous LowDensity Nozzle Flows, AI-2590-A-2, Cornell Aeronautical Lab., Inc., Buffalo, New York, June 1970.

[3] Chung, C. H., Kim, S. C., Stubbs, R. M., and De Witt, K. J., "DSMC and Continuum Analyses of Low-Density Nozzle Flow," AIAA Paper 93-0727, Jan. 1993.

[4] Bird, G. A., Molecular Gas Dynamics, Oxford University Press, London, 1976.

[5] Penko, P. F., Boyd, I. D., Meissner, D. L., and De Witt, K. J., "Pressure Measurements in a Low-Density Nozzle Plume for Code Verification," AIAA Paper 912110, June, 1991.

[6] Boyd, I. D., Penko, P. F., Meissner, D. L., and De Witt, K. J., "Experimental and Numerical Investigations of Low-Density Nozzle and Plume Flows of Nitrogen," AIAA J., Vol. 30, No. 10, 1992, pp. 2453-2461.

[7] Campbell, D. H., "Nozzle-Lip Effects on Argon Expansions Into the Plume Backflow," J. Spacecraft, Vol. 26, No. 4, 1989, pp. 285-292.

[8] Campbell, D. H., "Angular Variation of Flowfield Properties in Free Jet Expansions," Rarefied Gas Dynamics, edited by A. E. Beylich, VCH Press, Weinheim, 
Germany, 1991, pp. 1019-1024.

[9] Nelson, D. A. and Doo, Y. C., "Simulation of Multicomponent Nozzle Flows into a Vacuum," Progress in Astronautics and Aeronautics: Rarefied Gas Dynamics, edited by E. P. Muntz, D. P. Weaver, and D. H. Campbell, AIAA, Washington, DC, Vol. 116, 1989, pp. 340-351.

[10] Zelesnik, D., Dunn, T., Micci, M. M., and Long, L. N., " Numerical and Experimental Investigation of Low Reynolds Number Nozzle Flows," AIAA Paper 91-3558, September, 1991.

[11] Bird, G. A., "Monte Carlo Simulation in an Engineering Context," Progress in Astronautics and Aeronautics: Rarefied Gas Dynamics, edited by Sam S. Fisher, Vol. 74, Part I, AIAA, New York, 1981, pp. 239255.

[12] Bird, G. A., "The Perception of Numerical Methods in Rarefied Gas Dynamics," Progress in Astronautics and
Aeronautics: Rarefied Gas Dynamics, edited by E. P. Muntz, D. P. Weaver, and D. H. Campbell, AIAA, Washington, DC, Vol. 118, 1989, pp. 211-226.

[13] Chung, C. H., Kim, S. C., Stubbs, R. M., and De Witt, K. J., "Analysis of Plum Backflow Around a Nozzle Lip in a Nuclear Rocket," AIAA Paper 93-2497, June 1993.

[14] Chung, C. H., Kim, S. C., De Witt, K. J., and Nagamatsu, H. T., "DSMC Analysis of Hypersonic LowDensity Flows Around a Scramjet Inlet," AIAA Paper 932870, July 1993.

[15] Borgnakke, C. and Larsen, P. S., "Statistical Collision Models for Monte Carlo Simulation of Polyatomic Gas Mixture", J. Computational Physics, Vol. 18, 1975, pp. 405-420.

[16] Boyd, I. D., "Analysis of Rotational Nonequilibrium in Standing Shock Waves of Nitrogen", AIAA J., Vol. 28, No. 11, 1990, pp. 1997-1999.

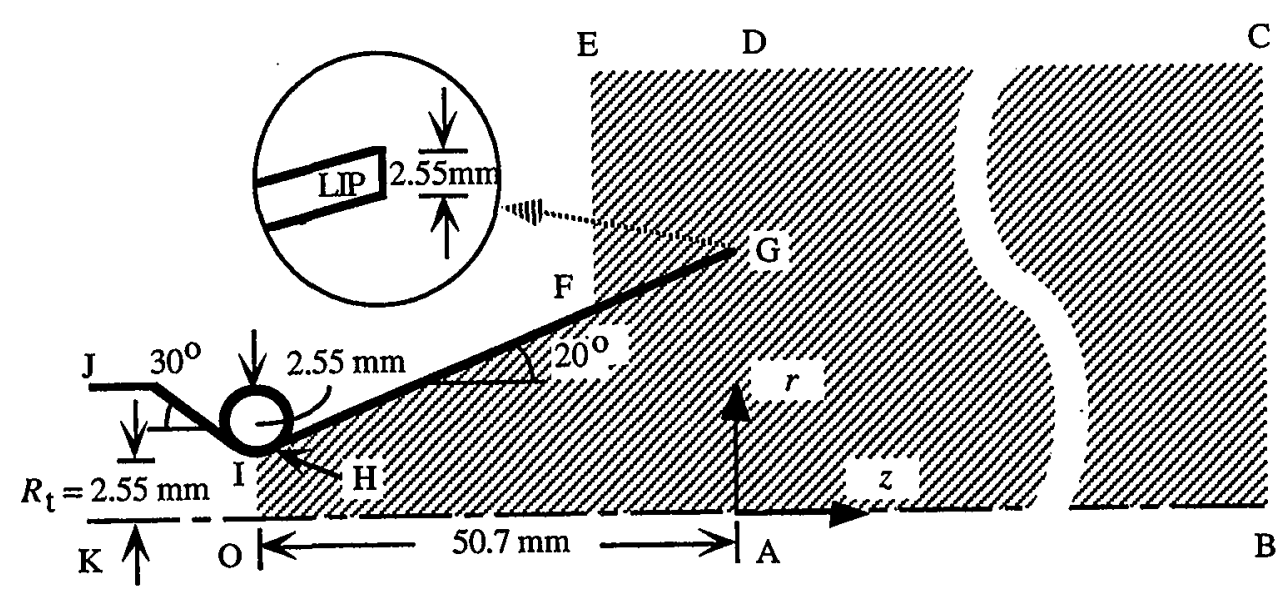

Fig. 1 Geometry of low-thrust nozzle.

Table 1 Flow conditions

\begin{tabular}{lll}
\hline & Case I & Case II \\
\hline Test gas & $\mathrm{N}_{2}$ & $\mathrm{~N}_{2}$ \\
Stagnation temperature, $T_{\mathrm{o}}$ & $300 \mathrm{~K}$ & $300 \mathrm{~K}$ \\
Stagnation pressure, $P_{\mathrm{o}}$ & $474 \mathrm{~Pa}$ & $1245 \mathrm{~Pa}$ \\
Ambient pressure, $P_{\mathrm{b}}$ & $1.5 \mathrm{~Pa}$ & $3.8 \mathrm{~Pa}$ \\
Wall temperature, $T_{\mathrm{W}}$ & $300 \mathrm{~K}$ & $300 \mathrm{~K}$ \\
Reynolds number, $R_{\mathrm{e}, \mathrm{t}}$ & 270 & 709 \\
Knudsen number, $K_{n}$ & $2.3 \times 10^{-3}$ & $8.8 \times 10^{-4}$ \\
\hline
\end{tabular}




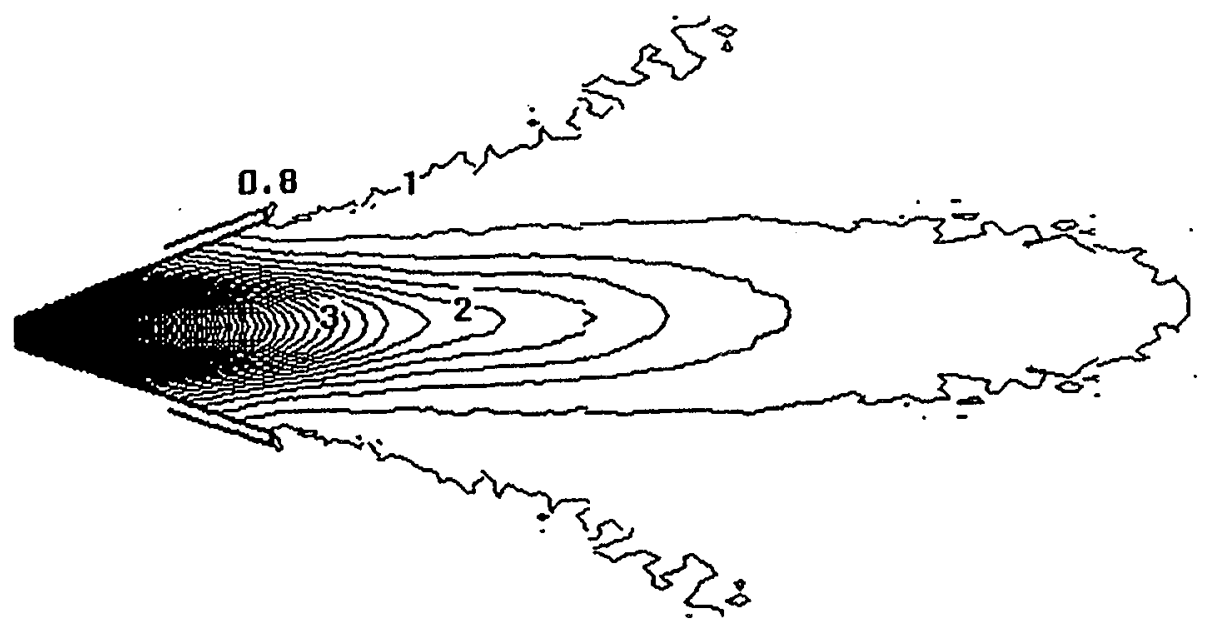

Fig. 2 Density contours for $P_{0}=474 \mathrm{~Pa}$ and $P_{0} / P_{b}=310$.

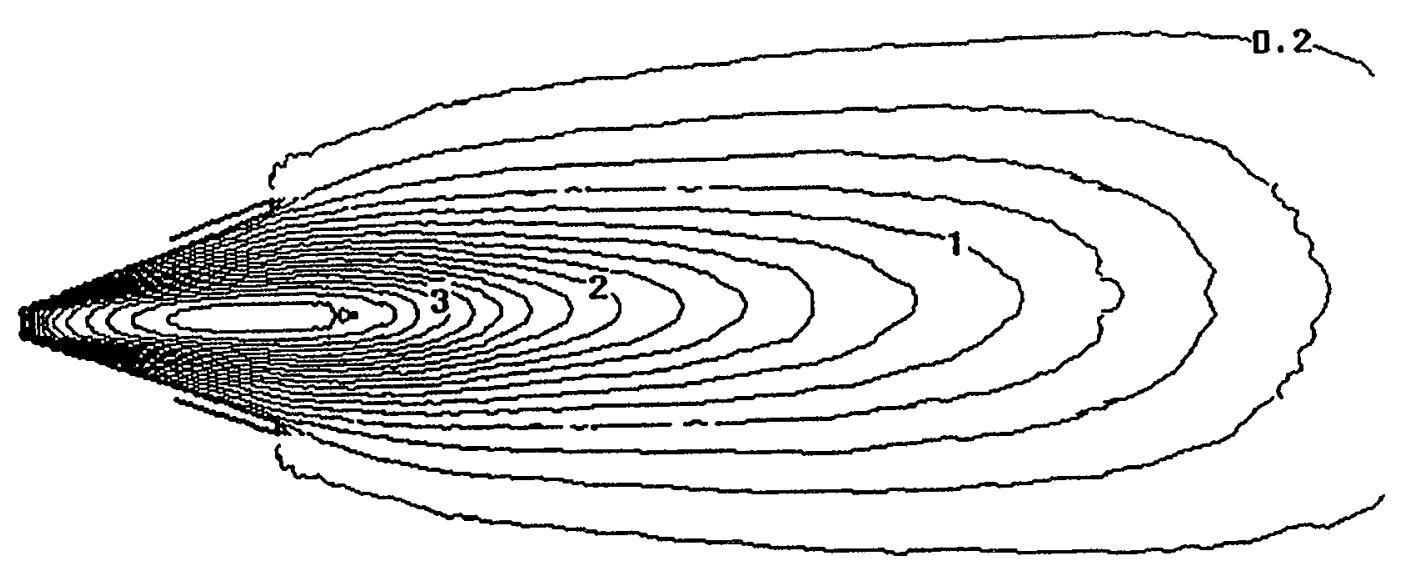

Fig. 3 Mach number contours for $P_{\mathrm{o}}=474 \mathrm{~Pa}$ and $P_{\mathrm{o}} / P_{\mathrm{b}}=310$.

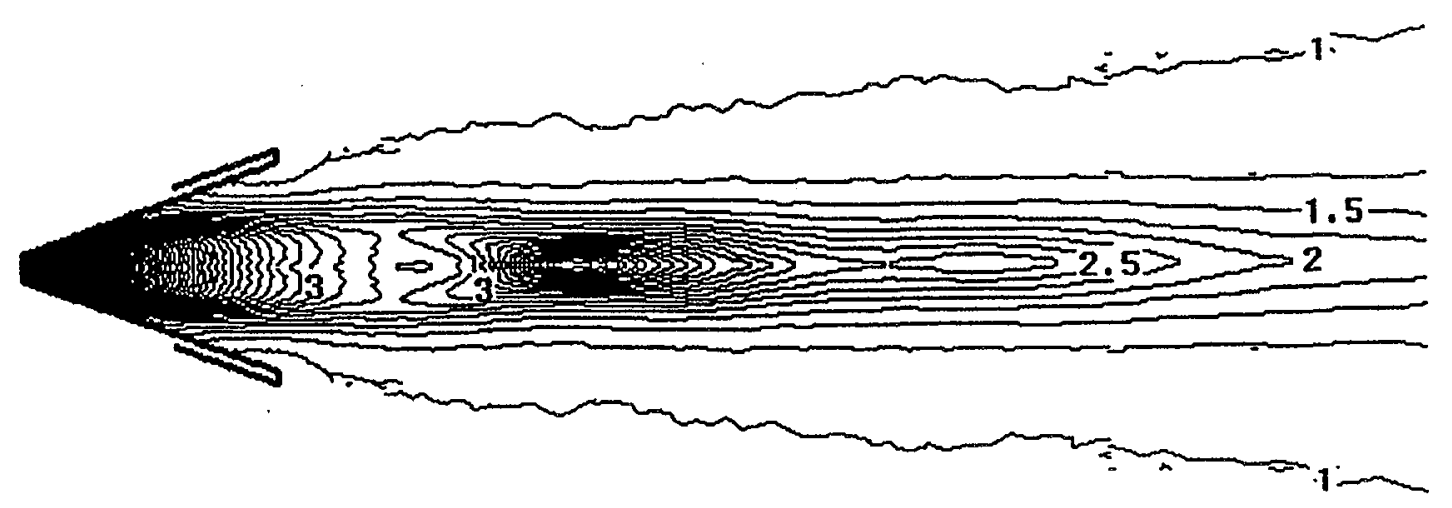

Fig. 4 Density contours for $P_{0}=1245 \mathrm{~Pa}$ and $P_{\mathrm{d}} / P_{\mathrm{b}}=325$. 


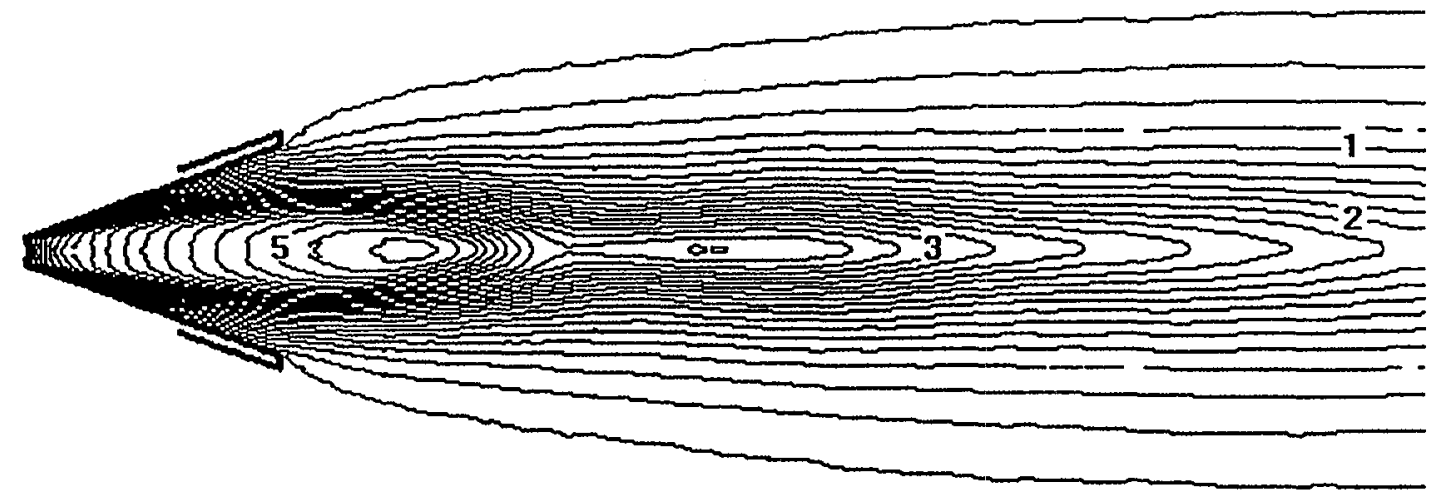

Fig. 5 Mach number contours for $P_{0}=1245 \mathrm{~Pa}$ and $\boldsymbol{P}_{\mathrm{o}} / P_{\mathrm{b}}=325$.

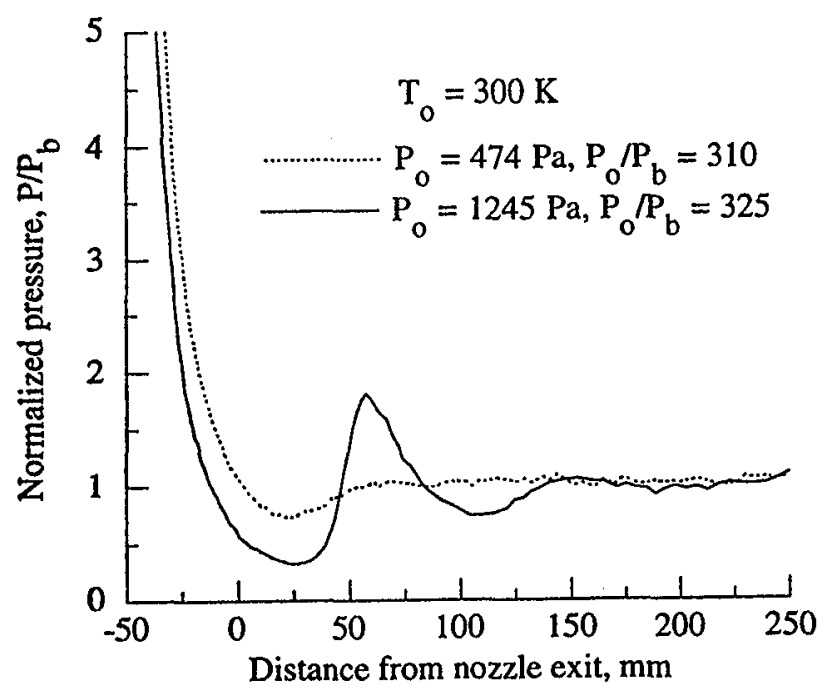

Fig. 6 Pressure distribution along the axis.

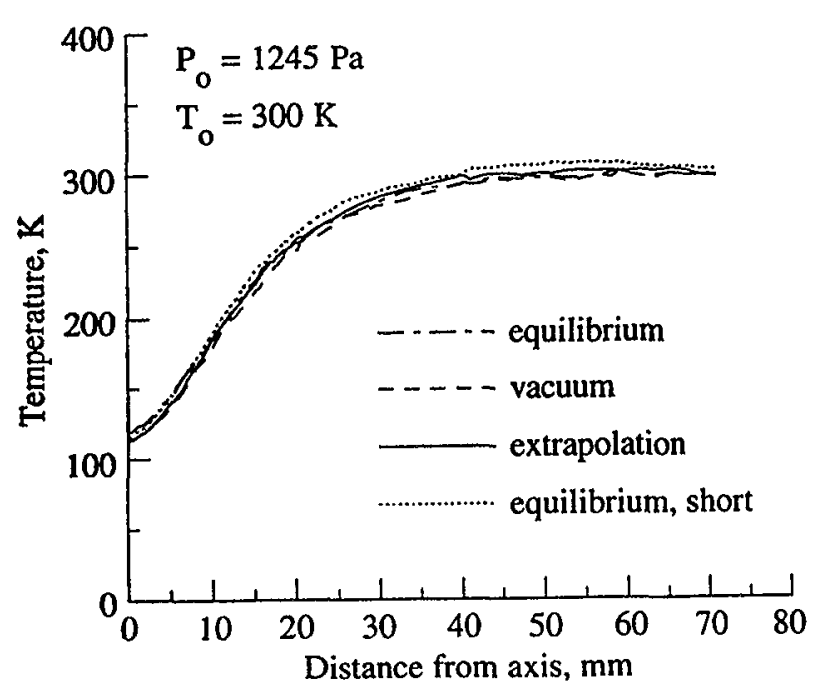

Fig. 8 Translational temperature distribution at $z=150 \mathrm{~mm}$.

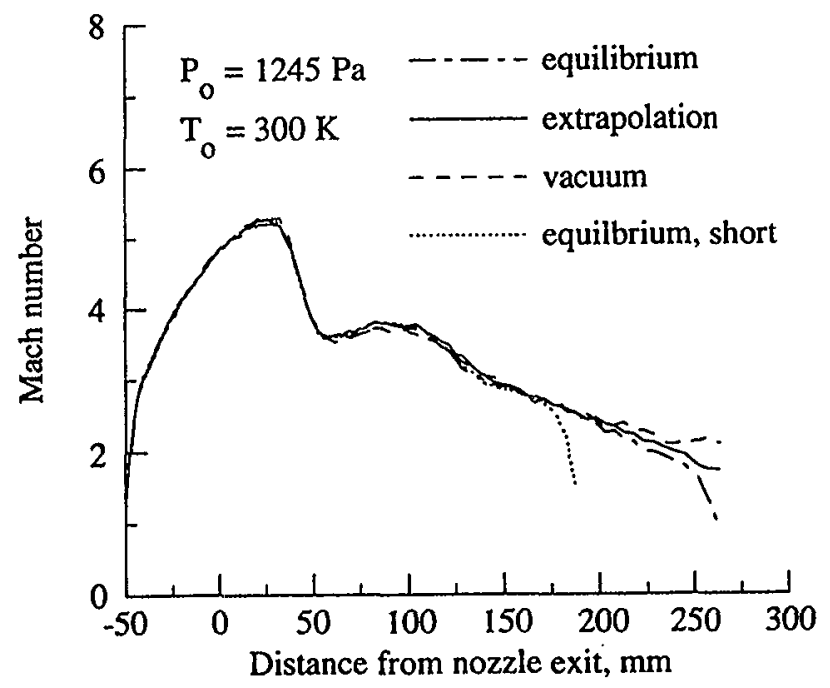

Fig. 7 Mach number distribution along the axis.

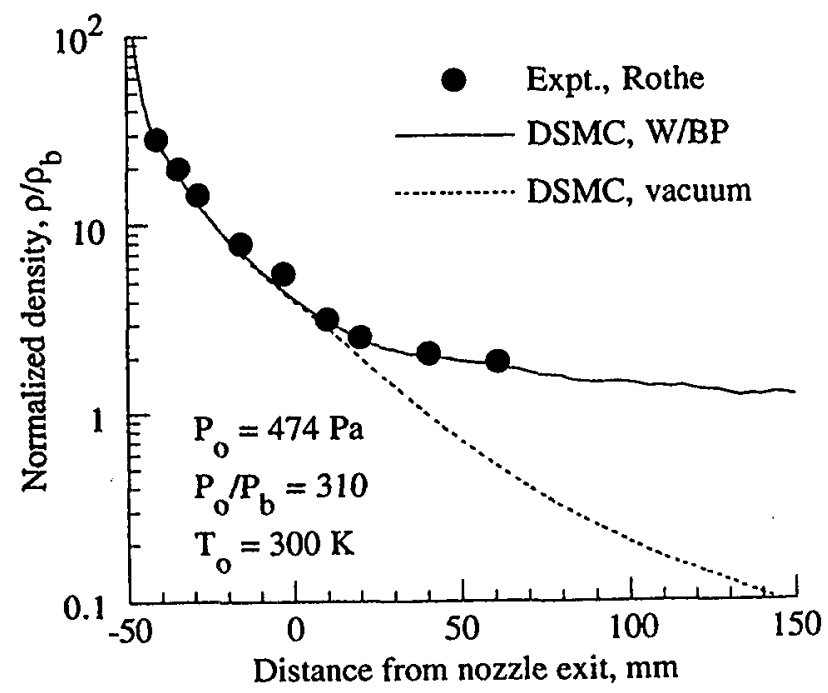

Fig. 9 Density distribution along the axis for $P_{0}=474 \mathrm{~Pa}$. 


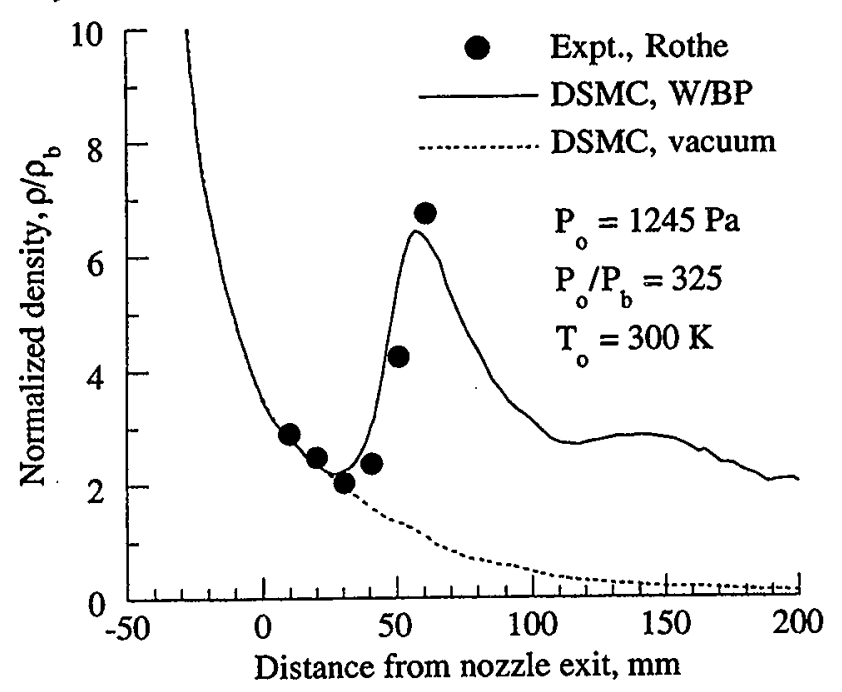

Fig. 10 Density distribution along the axis for $P_{0}=1245 \mathrm{~Pa}$.

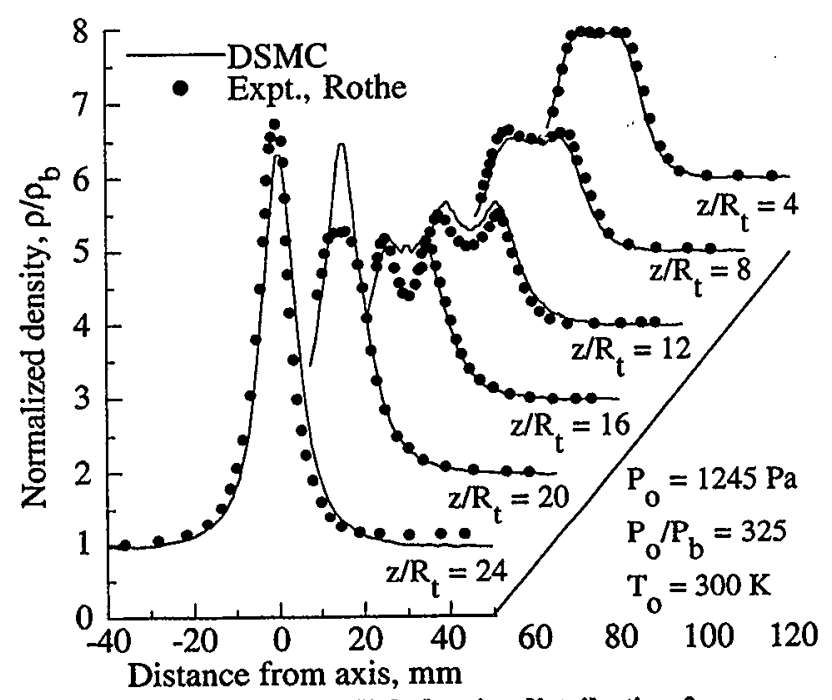

Fig. 12 Comparison of radial density distribution for $P_{0}=1245 \mathrm{~Pa}$.

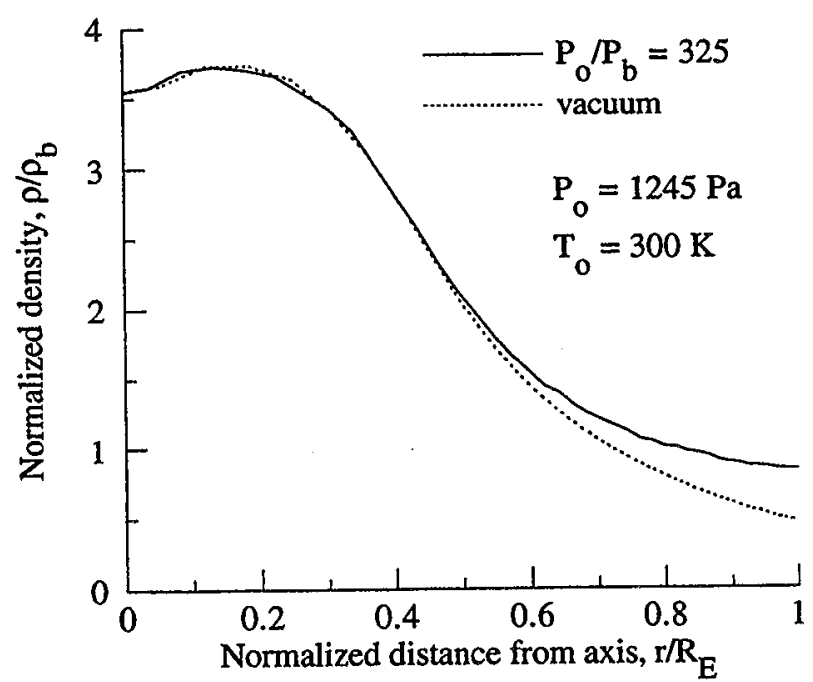

Fig. 14 Density distribution at exit plane for $P_{0}=1245 \mathrm{~Pa}$.

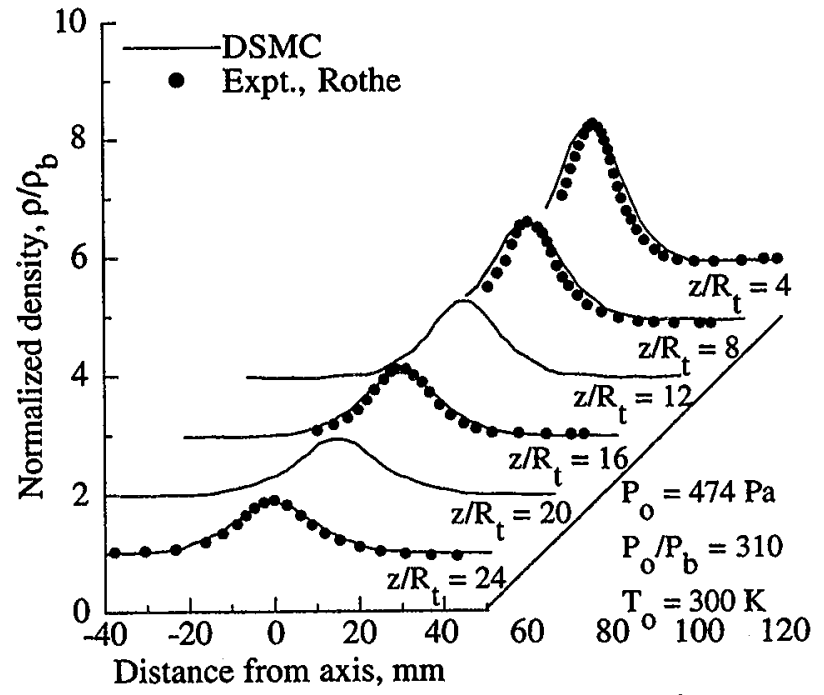

Fig. 11 Comparison of radial density distribution for $P_{0}=474 \mathrm{~Pa}$.

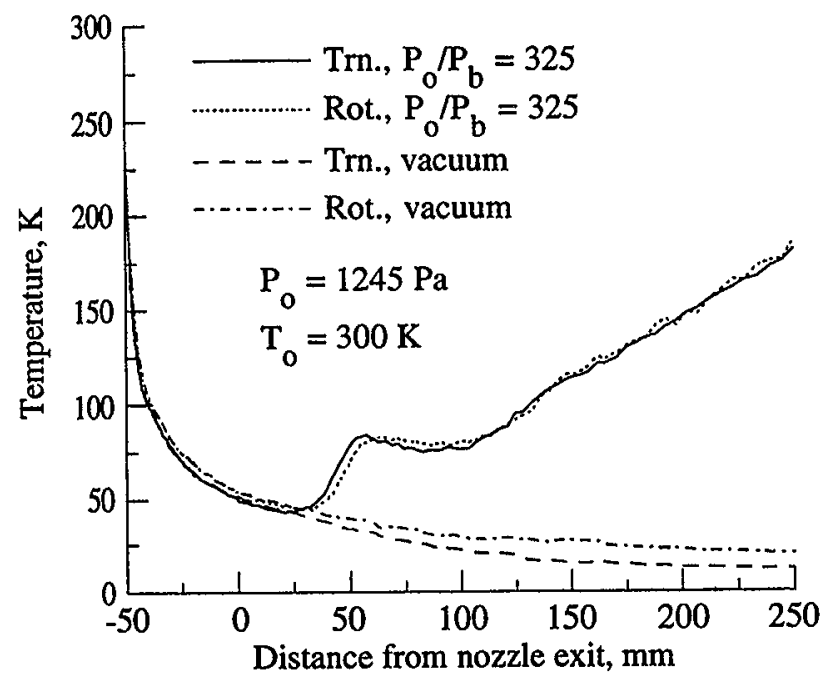

Fig. 13 Temperature distribution along the axis.

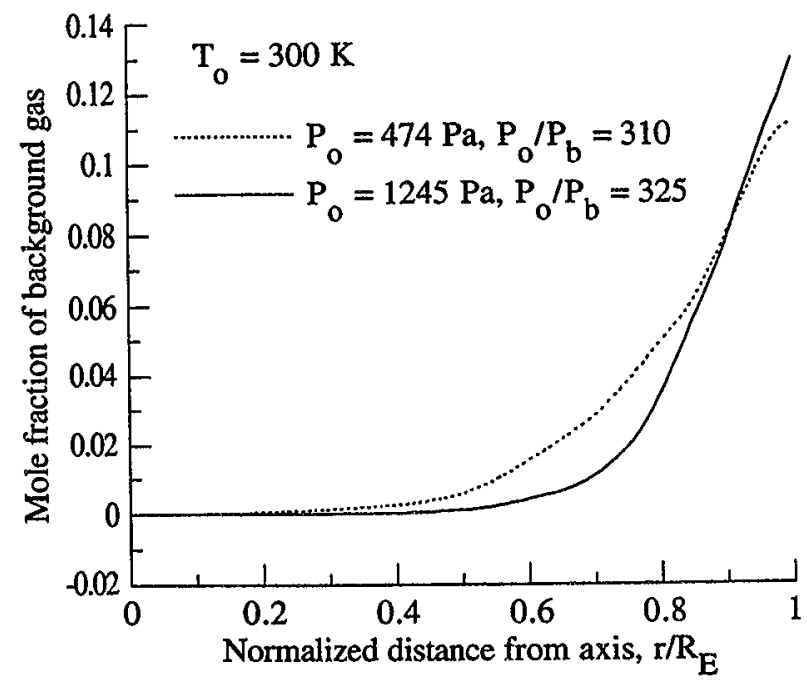

Fig. 15 Mole fraction of ambient gas at exit plane. 


\section{$\checkmark$}
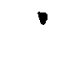

. 
Public reporting burden for this collection of information is estimated to average 1 hour per response, including the time for reviewing instructions, searching existing data sources, gathering and maintaining the data needed, and completing and reviewing the collection of information. Send comments regarding this burden estimate or any other aspect of this collection of information, including suggestions for reducing this burden, to Washington Headquarters Services, Directorate for Information Operations and Reports, 1215 Jefferson Davis Highway, Suite 1204, Artington, VA 22202-4302, and to the Office of Management and Budget, Paperwork Reduction Project (0704-0188), Washington, DC 20503.
1. AGENCY USE ONLY (Leave blank)
2. REPOAT DATE
February 1994
3. REPORT TYPE AND DATES COVERED
Funplomum

4. TITLE AND SUBTITLE

Simulation of Low-Density Nozzle Plumes in Non-Zero Ambient Pressures 6. AUTHOR(S)

WU-505-62-52

Chan-Hong Chung, Kenneth J. De Witt, Robert M. Stubbs, and Paul F. Penko

7. PERFORMING ORGANIZATION NAME(S) AND ADDRESS(ES)

National Aeronautics and Space Administration

Lewis Research Center

5. FUNDING NUMBERS

Cleveland, Ohio 44135-3191

8. PERFORMING ORGANIZATION REPORT NUMBER

E-8514

9. SPONSORING/MONITORING AGENCY NAME(S) AND ADDRESS(ES)

10. SPONSORING/MONITORING AGENCY REPORT NUMBER

National Aeronautics and Space Administration

Washington, D.C. 20546-0001

NASA TM-106501

AIAA-94-0357

11. SUPPLEMENTARY NOTES

Prepared for the 32nd Aerospace Sciences Meeting and Exhibit sponsored by the American Institute of Aeronautics and Astronautics, Reno, Nevada, January 10-13, 1994. Chan-Hong Chung, University of Toledo, Toledo, Ohio 43606 and NASA Resident Research Associate at Lewis Research Center (work funded by NASA Contract NCC3-171); Kenneth J. De Witt. The University of Toledo, Department of Chemical Engineering, Toledo, Ohio 43606; and Robert M. Stubbs and Paul F. Penko, NASA Lewis Research Center. Responsible person, Robert M. Stubbs, organization code 2670, (216) $433-6303$.

\begin{tabular}{l|l} 
12a. DISTRIBUTION/AVAILABILITY STATEMENT & 12b. DISTRIBUTION CODE
\end{tabular}

Unclassified-Unlimited

Subject Category 20

13. ABSTRACT (Maximum 200 words)

The direct simulation Monte-Carlo (DSMC) method was applied to the analysis of low-density nitrogen plumes exhausting from a small converging-diverging nozzle into finite ambient pressures. Two cases were considered that simulated actual test conditions in a vacuum facility. The numerical simulations readily captured the complicated flow structure of the overexpanded plumes adjusting to the finite ambient pressures, including Mach disks and barrelshaped shocks. The numerical simulations compared well to experimental data of Rothe.

Direct simulation; Monte-Carlo Method; Low-density flow; Non-zero ambient pressure

17. SECURITY CLASSIFICATION OF REPORT Unclassified
18. SECURITY CLASSIFICATION OF THIS PAGE Unclassified
19. SECURITY CLASSIFICATION OF ABSTRACT Unclassified 
National Aeronautics and

Space Administration

Lewis Research Center

21000 Brookpark Rd.

Cleveland, OH 44135-3191

Officlal Business

Penally for Privale Uso $\$ 300$

POSTMASTER: If Undellverable - Do Not Return

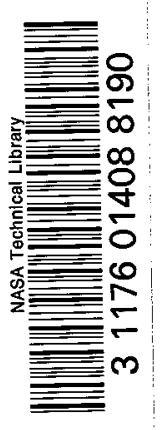

